\title{
Prevent hypotension and hypoxemia
}

David Johnson MD

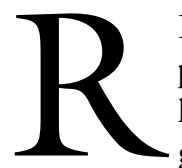

ECOMMENDATIONS by consultants to prevent intraoperative hypotension and hypoxemia in critically ill or high risk surgical patients are viewed by anesthesiologists as stating the obvious. However, even if hypotension and hypoxemia are avoided or aborted, standard monitoring of vital signs and arterial saturation does not always ensure the adequacy of tissue perfusion and subsequent prevention of organ failure. In this issue of the Canadian Journal of Anesthesia, Hong et al. ${ }^{1}$ report the effect of augmenting flow in a canine model of hemorrhagic shock and the effect of dopamine and dobutamine upon splanchnic perfusion as measured by gastric $\mathrm{PCO}_{2}$, calculated intramucosal $\mathrm{pH}(\mathrm{pHi})$, and indexes of oxygen delivery/consumption. Their letter reminds clinicians of the need for monitors to prognosticate outcome after injury. Ideally the selected monitor would allow clinicians to improve outcome after detection and correction of a physiological abnormality.

The widespread use of invasive cardiac monitoring reveals the association between indices of perfusion (cardiac output, systemic vascular resistance) and oxygen consumption in critically ill patients. Theoretically the adequacy of tissue oxygenation can be measured globally as an index of anaerobic metabolism or by measuring the tissue perfusion of single organs. It has been argued that tissue oxygenation measurement is unnecessary if adequate oxygen delivery could be assured. Oxygen delivery may help determine if a patient is demonstrating supply dependency and by simply increasing transport the relative insufficiency would be ameliorated. Critical values for adequate oxygen delivery are a cardiac index greater than 4.5 $\mathrm{L} \cdot \mathrm{min}^{-1} \cdot \mathrm{m}^{2}$, oxygen delivery index greater than 600 $\mathrm{mL} \cdot \mathrm{min}^{-1} \cdot \mathrm{m}^{2}$, and oxygen consumption greater than $170 \mathrm{~mL} \cdot \mathrm{min}^{-1} \cdot \mathrm{m}^{2} .^{2}$ Although many studies have demonstrated that survival is associated with attaining threshold critical values, attempts to enhance the hyperdynamic response with pharmacological agents (dobutamine, dopexamine) have not shown a consis- tent response in more than 18 randomized control trials. $^{2}$ The dependency of oxygen consumption upon delivered oxygen may simply be an artifact of measurement. ${ }^{3}$

If oxygen delivery is not a useful monitor, would a global measurement of inadequate tissue perfusion be better? Serum lactate has been used to monitor adequacy of tissue perfusion. Although prognosis is worse with elevated serum lactate, this is both a late and nonspecific finding. Lactate is a balance between production and metabolic elimination with the latter being probably more important. ${ }^{4}$ As such, it is not the ideal monitor.

The limitations of oxygen delivery and global tissue perfusion measurement as monitors have led to the clinical introduction of gastric tonometry in the early 1990's. Tonometry measures the gas tension in a saline or air filled silicone balloon which is semipermeable to gas and equilibrates with gastric or other viscous fluids. Although both oxygen and carbon dioxide can be measured, the theoretical index of adequate oxygen is unknown. Authors often report $\mathrm{pHi}$ rather than $\mathrm{PCO}_{2}$ which is simply calculated from the HendersonHasselbalch equation where $\mathrm{pHi}=6.1+\left(\log \left[\mathrm{HCO}_{3 \text { arte }}\right.\right.$ rial / constant $(0.03)$ * tonometer saline $\left.\mathrm{PCO}_{2}\right)$. An elevation of pHi may be reflective of accumulation of $\mathrm{CO}_{2}$ or as the buffered product of anaerobic hydrogen ion metabolism with both of these occurring due to inadequate perfusion. The divergence of arterial and intramucosal $\mathrm{pH}$ is reflective of specific gastric mucosal hypoperfusion.

Gastric tonometry has been touted to be an organ specific metabolic monitor of tissue hypoxia detecting an otherwise silent gut ischemia. Early reports of gastric tonometry in shock indicated that a critical level of less than 7.35 was associated with mortality and morbidity (and more prognostic than lactate, $\mathrm{O}_{2}$ delivery/consumption measurements, or physiological scores such as APACHE). ${ }^{5,6}$ As well, pHi has been used to predict success of separation from mechanical ventilation $^{7}$ and morbidity after major abdominal

From the Department of Medicine, Anesthesia, Community Medicine and Epidemiology, University of Saskatchewan, Saskatoon, Saskatchewan, Canada.

Address correspondence to: Dr. David Johnson, Department of Anesthesia, University of Saskatchewan, Saskatoon, Saskatchewan S7N 0W8, Canada. Phone: 306-655-1183; Fax: 306-655-8021; E-mail: cujec@V-ave.com 
surgery. ${ }^{8}$ Gastric tonometry has not been as predictive of morbidity post cardiopulmonary bypass ${ }^{9}$ or after aortic cross clamping. ${ }^{10}$

Two randomized studies have assessed the use of pHi to stratify patients and as a monitor of successful resuscitation. Therapy to increase oxygen delivery in critically ill patients with normal pHi (greater than 7.35 ) but not low pHi (less than 7.35) significantly increased survival in an unblinded study of 260 patients. In this study all groups received similar therapy (fluids, transfusion, dopamine, dobutamine). The control groups did not follow a standardized protocol and the high pHi control group had a unexpected high mortality resulting in a significant difference with the treatment group. ${ }^{5}$ In a randomized study of 210 critically ill patients treated with colloids then dobutamine to increase $\mathrm{O}_{2}$ delivery if $\mathrm{pHi}$ was less than 7.35 , mortality was not different between groups nor was pHi consistently elevated with standardized treatment. ${ }^{11}$ These randomized clinical trial results require a reevaluation of gastric tonometry as a useful monitor to guide therapy.

Tonometry can be considered to be a long time constant, gastric capnogram. Intramural $\mathrm{PCO}_{2}$ does not correlate well with direct measurements of intramural hypoxia. ${ }^{12} \mathrm{PCO}_{2}$ production seen with hypoperfusion is not solely or simply related to hypoxic anaerobic metabolism. The ability to assess tissue hypoxia from tissue hypercarbia is not used in any other organ. So, the lack of correlation with direct measurements of mucosal $\mathrm{O}_{2}$ is not surprising. ${ }^{12}$ As well, similar to any technique, technical limitations are present. Gastric secretion of hydrogen ions increases $\mathrm{CO}_{2}$ produced via bicarbonate buffering and is affected by feeding. ${ }^{12}$ Equilibration of $\mathrm{CO}_{2}$ is slow (30 to $60 \mathrm{~min}$ ) and wide rapid swings in hemodynamic status are difficult to monitor. Gastric $\mathrm{CO}_{2}$ is affected by systemic $\mathrm{CO}_{2}$ and may yield misleading data in setting of respiratory acidosis or alkalosis. ${ }^{12}$ Arterial bicarbonate may not be identical to intramucosal bicarbonate which makes $\mathrm{PCO}_{2}$ or the gap between arterial and tonometric $\mathrm{CO}_{2}$ a better monitor than pHi. ${ }^{12}$

So what conclusions can we draw from our study? We know from this study and others ${ }^{13}$ that prompt and adequate replacement of volume after hemorrhagic shock does not prevent the decrease in $\mathrm{pHi}$ in the first few hours postresuscitation using a canine shock model. As well, volume expansion does not increase pHi in hypovolemic patients with sepsis ${ }^{4} \mathrm{In}$ accordance with this study, a synthesis of ten clinical and experimental studies also concluded that dopamine was less likely and dobutamine most likely to increase pHi in shock. ${ }^{15}$ In general, therapies that vasodilate (dobutamine) increase $\mathrm{pHi}$ whereas increases in $\mathrm{O}_{2}$ delivery (red blood cell transfusions, volume, dopamine) do not improve $\mathrm{pHi}^{1{ }^{16}}$

More generally, it is too simple to expect that simply stopping the hemorrhage and priming the vasculature with lost fluid will restore homeostasis and ensure good outcome. The right mix of blood, colloids, or crystalloids; the right amount of fluid to administer; or ensuring sufficient flow may only partially restore homeostasis. Our present resuscitation monitors focus on vascular anatomy (flow and pressure) or a simple quantification of vessel content $\left(\mathrm{O}_{2}, \mathrm{CO}_{2}\right.$, cells, clotting factors). Shock induced by hemorrhage or other types of injury impact upon coagulation, inflammation, immunity, and cellular metabolism. As well, genetic pleomorphism may mean that one strategy does not fit all patients. The astute clinician must understand both the value and limitations of the present toolkit of monitors/therapy and be willing to add new tried and verified tools of the trade.

The clinical lessons we can draw from this study and work by other investigators are that:

1) profound hemorrhage may result in subsequent morbidity such as multiple system organ failure in some patients even if the blood loss and hypotension are promptly reversed;

2) gastric tonometry is a single end organ measure of perfusion with some prognostic value in shock but a poor monitor to guide or stratify therapy;

3 ) early resuscitation during shock may also require as yet unknown therapy that halts the physiological and biochemical processes that cause subsequent morbidity;

4) vasoactive drugs like dopamine or dobutamine are unlikely to be particularly useful as early preventative therapies than can abort the physiological and biochemical processes causing subsequent morbidity;

5 ) the monitors that guide future resuscitative therapy in hemorrhagic shock may not just be physiological (blood pressure, cardiac output, oxygen delivery) but be based on the biochemical processes initiated with shock. 


\section{Prévenir l'hypotension et l'hypoxémie}

Les recommandations des médecins-conseils sur la prévention de l'hypotension et de l'hypoxémie peropératoires chez les patients gravement malades ou chez les opérés à haut risque semblent aller de soi pour les anesthésiologistes. Cependant, même si l'hypotension et l'hypoxémie sont évitées ou supprimées, le monitorage habituel des signes vitaux et de la saturation artérielle n'assure pas toujours la perfusion tissulaire adéquate et la prévention d'une défaillance organique subséquente. Dans le présent numéro du Journal, Hong et coll. ${ }^{1}$ décrivent l'effet de l'augmentation du débit sanguin chez un modèle canin de choc hémorragique et l'effet de la dopamine et de la dobutamine sur la perfusion splanchnique définie par la mesure de la $\mathrm{PCO}_{2}$ gastrique, le calcul du $\mathrm{pH}$ intramuqueux (Phi) et les index d'apport/consommation d'oxygène. L'étude nous rappelle la nécessité des moniteurs pour prédire l'issue d'une maladie. Idéalement, le moniteur choisi permet d'améliorer l'évolution après la détection et la correction d'une anomalie physiologique.

L'utilisation largement répandue du monitorage cardiaque effractif montre un lien entre les indices de perfusion (débit cardiaque, résistance vasculaire générale) et la consommation d'oxygène chez les grands malades. En théorie, le caractère adéquat de l'oxygénation tissulaire peut être mesuré globalement par un index du métabolisme anaérobique ou par la mesure de la perfusion tissulaire d'organes individuels. On a avancé que la mesure de l'oxygénation tissulaire n'était pas nécessaire si un apport d'oxygène adéquate pouvait être assuré. La détermination de l'apport d'oxygène peut indiquer si le patient en reçoit suffisamment et permettre, par une simple augmentation, de remédier au manque relatif. Un apport d'oxygène suffisant est représenté par les valeurs critiques suivantes : un index cardiaque plus grand que 4,5 $\mathrm{L} \cdot \mathrm{min}^{-1} \cdot \mathrm{m}^{2}$, un apport d'oxygène plus grand que 600 $\mathrm{mL} \cdot \mathrm{min}^{-1} \cdot \mathrm{m}^{2}$ et une consommation d'oxygène plus élevée que $170 \mathrm{~mL} \cdot \mathrm{min}^{-1} \cdot \mathrm{m}^{2} .^{2}$ Nombre d'études ont démontré déjà que la survie dépend de l'atteinte des valeurs seuil critiques, mais les résultats de 18 essais contrôlés et randomisés, ${ }^{2}$ réalisés dans le but de stimuler la réponse hyperdynamique avec des agents pharmacologiques (dobutamine, dopexamine), n'ont pas été constants. Le rapport de dépendance entre la consommation et l'apport d'oxygène ne pourrait être qu'un artéfact de la mesure. ${ }^{3}$

Si l'apport d'oxygène n'est pas un indicateur utile, une mesure globale de l'insuffisance de la perfusion tissulaire serait-elle meilleure ? Le lactate sérique a été utilisé pour surveiller la qualité de la perfusion tissulaire. Bien que le pronostic soit plus sombre avec un lactate sérique élevé, c'est un résultat à la fois tardif et non spécifique. Le lactate exprime le bilan entre la production et l'élimination métabolique, cette dernière étant probablement plus importante. ${ }^{4}$ À ce titre, il ne constitue par un indicateur idéal.

Les limites des mesures de l'apport d'oxygène et de la perfusion tissulaire globale, comme instruments de surveillance, ont conduit à l'introduction clinique de la tonométrie gastrique au début de la décennie 1990. La tonométrie mesure la tension d'un gaz à l'intérieur d'une solution saline ou d'un ballon de silicone rempli d'air. Ce ballon est semi-perméable au gaz et son contenu s'équilibre avec les liquides gastrique ou autres liquides visqueux. Bien que l'on puisse mesurer l'oxygène et le gaz carbonique, on ne connaît pas encore l'index théorique d'un apport d'oxygène suffisant. Les auteurs mentionnent souvent le pHi plutôt que la $\mathrm{PCO}_{2}$ qui est simplement calculée à partir de l'équation de Henderson-Hasselbalch où le $\mathrm{pHi}=6,1$ $+\left(\log \left[\mathrm{HCO}_{3 \text { artériel }}\right] /\right.$ constante $(0,03)^{*} \mathrm{PCO}_{2}$ saline tonométrique). Une hausse du pHi peut refléter l'accumulation de $\mathrm{CO}_{2}$ ou le produit réduit du métabolisme anaérobique de l'ion d'hydrogène, ces deux événements survenant à la suite d'une perfusion inadéquate. La divergence entre le $\mathrm{pH}$ artériel et intramuqueux traduit l'hypoperfusion spécifique de la muqueuse gastrique.

La tonométrie gastrique a été reconnue comme moyen de surveillance métabolique d'hypoxie tissulaire spécifique pouvant détecter une ischémie intestinale autrement silencieuse. Les premières données sur la tonométrie gastrique réalisée chez des patients en choc montraient qu'un niveau critique de moins de 7,35 était associé à la mortalité et la morbidité. C'est une technique plus prédictive que la mesure du lactate et de l'apport/la consommation d' $\mathrm{O}_{2}$ ou que les scores physiologiques, type APACHE).$^{5,6} \mathrm{Le} \mathrm{pHi}$ a aussi été utilisé pour prédire la réussite du sevrage de la ventilation mécanique ${ }^{7}$ et la morbidité après une intervention chirurgicale abdominale importante. ${ }^{8} \mathrm{La}$ tonométrie gastrique n'est toutefois pas aussi prédictive de la morbidité après la circulation extracorporelle $^{9}$ ou le clampage de la crosse aortique. ${ }^{10}$

On a étudié, au cours de deux études randomisées, l'utilisation du pHi pour répartir les groupes de patients (mode stratifié) et contrôler la réanimation. 
Le traitement visant à accroître l'apport en oxygène chez de grands malades qui présentent un $\mathrm{pHi}$ normal (plus élevé que 7,35 ), non un faible pHi (plus bas que 7,35 ), augmente la survie comme le démontre une étude ouverte de 260 patients. Tous ont reçu un traitement identique (liquides, transfusion, dopamine, dobutamine). Ceux des groupes témoins n'ont pas été soumis à un protocole normalisé et les patients témoins à pHi élevé ont présenté un important taux de mortalité inattendu, ce qui a donné lieu à une différence significative avec les patients du groupe expérimental. ${ }^{5}$ Dans une étude randomisée de 210 grands malades traités avec des colloïdes, puis de la dobutamine pour augmenter l'apport en $\mathrm{O}_{2}$ si le pHi était plus bas que 7,35 , la mortalité n'a pas présenté de différence intergroupe et le pHi n'est pas demeuré constamment élevé avec le traitement normalisé. ${ }^{11}$ Les résultats de ces essais cliniques randomisés commandent donc une réévaluation de la pertinence de la tonométrie gastrique comme moyen d'orienter le traitement.

On peut voir la tonométrie comme un capnogramme gastrique constant et prolongé. Il y a peu de corrélation entre la $\mathrm{PCO}_{2}$ intramurale et les mesures directes de l'hypoxie intramurale. ${ }^{12}$ La production de $\mathrm{PCO}_{2}$, observée avec l'hypoperfusion, n'est pas uniquement et simplement reliée au métabolisme hypoxique anaérobique. La capacité de détecter l'hypoxie tissulaire à l'aide de l'hypercapnie tissulaire n'est utilisée pour aucun autre organe. Donc, l'absence de corrélation avec les mesures directes de $\mathrm{l}^{\prime} \mathrm{O}_{2}$ de la muqueuse était à prévoir. ${ }^{12}$ De plus, c'est une technique, comme toutes les autres, qui a ses limites. La sécrétion gastrique d'ions d'hydrogène augmente le $\mathrm{CO}_{2}$ produit par le tamponnage bicarbonate et subit les effets de l'alimentation. ${ }^{12}$ L'équilibration du $\mathrm{CO}_{2}$ est lente ( 30 à $60 \mathrm{~min}$ ), et les changements rapides et importants de l'état hémodynamique sont difficiles à surveiller. $\mathrm{Le} \mathrm{CO}_{2}$ gastrique subit l'effet du $\mathrm{CO}_{2}$ systémique et peut fournir des données erronées s'il y a une acidose ou une alcalose respiratoire. ${ }^{12}$ Le bicarbonate artériel pourrait être différent du bicarbonate intramuqueux, ce qui permet de faire de la $\mathrm{PCO}_{2}$ ou de l'écart entre le $\mathrm{CO}_{2}$ artériel et tonométrique un meilleur moniteur que le $\mathrm{pHi}^{1}{ }^{2}$

Que pouvons-nous conclure de notre étude ? Nous avons appris, de la présente étude et d'autres, ${ }^{13}$ que le remplissage vasculaire rapide et suffisant, fait à la suite d'un choc hémorragique, ne prévient pas la baisse du pHi pendant les premières heures qui suivent la réanimation chez un modèle de choc canin. Aussi, l'expansion volumique n'augmente pas le pHi chez les patients hypovolémiques atteints de septicémie. ${ }^{14}$
Conformément à cette étude, une synthèse de dix études expérimentales et cliniques a aussi conclu que la dopamine est moins susceptible d'augmenter le pHi pendant le choc, ${ }^{15}$ mais que la dobutamine l'est davantage. En général, les traitements vasodilatateurs (dobutamine) augmentent le pHi tandis qu'une augmentation de l'apport d' $\mathrm{O}_{2}$ (transfusions de globules rouges, expansion volumique, dopamine) n'améliore pas le $\mathrm{pHi}^{16}$

De façon plus générale, il est trop simple d'espérer que le seul fait de stopper l'hémorragie et de procéder au remplissage vasculaire puisse restaurer l'homéostase et assurer une évolution positive. La combinaison appropriée de sang, colloïdes ou cristalloïdes, la quantité suffisante de liquide à administrer ou le maintien d'un débit adéquat peuvent restaurer l'homéostase de façon partielle seulement. La fonction des moniteurs de réanimation actuels est centrée sur l'anatomie (débit et pression) ou sur une simple quantification du contenu vasculaire $\left(\mathrm{O}_{2}, \mathrm{CO}_{2}\right.$, cellules, facteurs de coagulation). Le choc induit par l'hémorragie ou d'autres types de lésions agit sur la coagulation, l'inflammation, l'immunité et le métabolisme cellulaire. Aussi, le polymorphisme génétique pourrait signifier qu'une stratégie ne convient pas à tous les patients. Le clinicien bien avisé doit connaître la valeur et les limites des thérapies et des moniteurs qui font actuellement partie de l'arsenal de notre spécialité et consentir à y ajouter de nouveaux outils qui ont été essayés et vérifiés.

Les leçons cliniques à tirer de l'ensemble des études examinées sont que :

1) Une hémorragie profonde peut causer une mor bidité ultérieure, comme une défaillance multiviscérale, chez certains patients même si la perte sanguine et l'hypotension sont rapidement renversées.

2) La tonométrie gastrique est une mesure unique de la perfusion d'un organe cible, qui présente une certaine valeur pronostique pendant un choc, mais un moyen pauvre de guider ou de doser le traitement.

3) La réanimation précoce pendant le choc peut aussi exiger un traitement encore inconnu à ce jour, capable d'interrompre le processus physiologique et biochimique responsable de la morbidité ultérieure.

4) Les médicaments vasoactifs comme la dopamine ou la dobutamine ne semblent pas particulièrement utiles comme traitements préventifs précoces permettant d'interrompre le processus physiologique et biochimique responsable de la morbidité ultérieure. 
5) Les moniteurs qui, à l'avenir, vont guider la réanimation en cas de choc hémorragique ne seront peut-être pas seulement physiologiques (tension artérielle, débit cardiaque, apport d'oxygène), mais conçus pour informer sur le processus biochimique déclenché par le choc.

\section{References}

1 Hong SJ, Kim JB, Chun JY, Lee HJ, Sung CH, Moon $S H$. Dobutamine, not dopamine, improves gastric intramucosal $\mathrm{pH}$ during resuscitation from hemorrhagic shock in dogs (Letter). Can J Anesth 2002; 49: 214.

2 Boyd $O$, Hayes $M$. The oxygen trail: the goal. Br Med Bull 1999; 55: 125-39 .

3 Matuschak GM. Supranormal oxygen delivery in critical illness. New Horiz 1997; 5: 233-8.

4 Brown SD, Gutierrez G Does gastric tonometry work? Yes. Crit Care Clin 1996; 12: 569-855.

5 Guttierez G, Palizas F, Doglio G, et al. Gastric intramucosal $\mathrm{pH}$ as a theraputic index of tissue oxygenation in critically ill patients. Lancet 1992; 339: 195-9.

6 Maynard N, Bihari D, Beale R, et al. Assessment of splanchnic oxygenation by gastric tonometry in patients with acute circulatory failure. JAMA 1993; 270: 1203-10.

7 Bocquillon N, Mathieu D, Neviere R, Lefebvre N, Marechal X, Wattel F. Gastric mucosal ph and blood flow during weaning from mechanical ventilation in patients with chronic obstructive pulmonary disease. Am J Respir Crit Care Med 1999; 160: 1555-61.

8 Theodoropoulos G, Lloyd LR, Cousins G, Pieper D. Intraoperative and early postoperative gastric intramucosal $\mathrm{pH}$ predicts morbidity and mortality after major abdominal sugery. Am Surg 2001; 67: 303-8.

9 Bams JL, Mariani MA, Groeneveld AB. Predicting outcome after cardiac surgery: comparison of global haemodynamic and tonometric variables. $\mathrm{Br} J$ Anaesth 1999; 82: 33-7.

10 Geelkerken RH, Zwijsen JH, van Baalen JM, Harinck $H I$, Hoffmann WH, van Bockel JH. Operations that require supracoeliac aortic cross-clamping:perioperative monitoring with gastric tonometry. Eur J Surg 1999; 165: 15-20.

11 Gomersall CD, Joynt GM, Freebairn RC, Hung V, Buckley TA, Oh TE. Resuscitation of critically ill patients based on the results of gastric tonometry: a prospective, randomized, controlled trial. Crit Care Med 2000; 28: 607-14.

12 Benjamin E, Oropello JM. Does gastric tonometry work? No. Crit Care Clin 1996; 12: 587-601.

13 Ond L, Kruse JAProgressive gastric intramucosal acidosis follows resuscitation from hemorrhagic shock. Shock 1996; 6: 61-5.
14 Forrest DM, Baigorri F, Chittock DR, Spinelli JJ, Russell $J A$. Volume expansion using pentastarch does not change gastric-arterial CO2 gradient or gastric intramucosal $\mathrm{pH}$ in patients who sepsis syndrome. Crit Care Med 2000; 28: 2254-8.

15 Silva E, DeBacker D, Creteur J, Vincent JL. Effects of vasoactive drugs on gastric intramucosal $\mathrm{pH}$. Crit Care Med 1998; 26: 1749-58.

16 Silverman HJ, Tuma P. Gastric tonometry in patients with sepsis. Effects of dobutamine infusions and packed red blood cell transfusions. Chest 1992; 102: 184-8. 